\title{
C-Reactive Protein/Albumin and Neutrophil/Albumin Ratios as Novel Inflammatory Markers in Patients with Schizophrenia
}

\author{
Yasin Hasan Balcioglu ${ }^{1,2} \bowtie$ and Simge Seren Kirlioglu ${ }^{1}$ \\ 1'Department of Psychiatry, Bakirkoy Prof Mazhar Osman Training and Research Hospital for Psychiatry, Neurology, and Neurosurgery, Istanbul, Turkey \\ ${ }^{2}$ Department of Medical Sciences, Institute of Forensic Sciences and Legal Medicine, Istanbul University-Cerrahpasa, Istanbul, Turkey
}

Objective Peripheral biomarker studies in schizophrenia are insufficient to correspond to whether inflammatory markers are trait- or state-related. The main objective of this study was to compare novel biomarkers C-reactive protein/albumin ratio (CAR), neutrophil/albumin ratio (NAR), and complete blood count-derived inflammatory markers; neutrophil/lymphocyte ratio (NLR), platelet/lymphocyte ratio (PLR), monocyte/lymphocyte ratio (MLR), red-cell distribution width (RDW), and mean platelet volume (MPV) between patients with acutely exacerbated and remitted schizophrenia and healthy controls.

Methods Anonymous data of a total of 618 patients with schizophrenia (179 in remission, 439 with acute exacerbation) and 445 psychiatrically and medically healthy subjects admitted to outpatient units were included. One-way ANOVA with Tukey's HSD post-hoc test, Pearson's correlation test, receiver operating characteristic analysis, and binomial logistic regression analysis were performed.

Results CAR, NAR, NLR, PLR, MLR, RDW, MPV values were found higher in patients with schizophrenia than in healthy subjects. Except for NAR ( $\mathrm{p}=0.007$ ), none of the markers differed between acute exacerbation and remission. As a cut-off value of CAR, 0.388 differentiated patients with schizophrenia from controls (sensitivity $81 \%$, specificity $81 \%$ ). CAR, NAR, and MPV significantly predicted the diagnosis of schizophrenia.

Conclusion CAR and NAR are reliable biomarkers of inflammation and a combination of inflammatory markers including CAR and NAR could be used to reflect the increased inflammatory status in schizophrenia, regardless of relapse or remission.

Psychiatry Investig 2020;17(9):902-910

Key Words C-reactive protein-albumin ratio, Schizophrenia, Inflammation, Relapse, Remission, Trait markers.

\section{INTRODUCTION}

Ongoing tireless research effort has been dedicated to elucidating the neurobiologic underpinnings of the etiology of schizophrenia for more than a half century. There has been tremendous renewed interest in the inflammation hypothesis of schizophrenia over the last two decades. The complex interactions between altered immune signaling and the brain were postulated in the development of schizophrenia., ${ }^{1,2}$ This allegation came from the striking findings of elaborative studies that showed increased pro-inflammatory cytokine and decreased

\footnotetext{
Received: May 17, 2020 Revised: July 3, 2020

Accepted: July 7, 2020

$\triangle$ Correspondence: Yasin Hasan Balcioglu, MD, PhD

Department of Psychiatry, Bakirkoy Prof Mazhar Osman Training and Research Hospital for Psychiatry, Neurology, and Neurosurgery, Istanbul 34147, Turkey

Tel: +90 21240915 15, Fax: +902124091590

E-mail: yhasanbalcioglu@gmail.com

(a) This is an Open Access article distributed under the terms of the Creative Commons Attribution Non-Commercial License (https://creativecommons.org/licenses/by$\mathrm{nc} / 4.0$ ) which permits unrestricted non-commercial use, distribution, and reproduction in any medium, provided the original work is properly cited.
}

anti-inflammatory cytokine plasma levels, ${ }^{3}$ up-regulated inflammatory gene expression, ${ }^{4}$ evidence of inflammation in certain cortical areas in post-mortem brains, ${ }^{5}$ a higher prevalence of autoimmune disorder comorbidity, ${ }^{6}$ and symptomatologic improvement with anti-inflammatory drugs, ${ }^{7}$ in schizophrenia. Inflammation is a complex mechanism based on multiple biological pathways and closely linked to oxidative stress. Inflammatory processes both directly and indirectly interact with the central nervous system and result in neurodegeneration, microglial activation, dysfunction of hypothalamic-pituitary-adrenal (HPA) axis, disrupted glutamate metabolism and altered neurotransmission, all of which have been postulated to be involved in schizophrenia etiopathogenesis. ${ }^{8}$

C-reactive protein (CRP) and albumin are acute phase reactants that respond to the systemic inflammation. ${ }^{9} \mathrm{CRP}$ is a well-documented circulating marker for systemic inflammation, ${ }^{10}$ and albumin is a negative acute-phase protein, serum levels of which are down-regulated in response to an inflammatory state. ${ }^{11}$ Unlike proinflammatory cytokines, both CRP 
and albumin have longer half-lives and are easier to measure in a blood screening. Increased plasma CRP, ${ }^{12}$ and decreased serum albumin levels ${ }^{13,14}$ have been shown in patients with schizophrenia in comparison with healthy controls. However, the CRP/albumin ratio (CAR) has been suggested to be a better indicator of an inflammatory response than CRP or albumin alone. ${ }^{15} \mathrm{CAR}$ has been proposed to reflect enhanced inflammatory tonus and increased levels of this biomarker are suggestive for poor prognosis and worse treatment outcomes in patients with systemic illnesses. ${ }^{15,16}$ Previously, CAR has been studied in several neurological illnesses including Parkinson's disease, migraine, and restless leg syndrome, and these studies have suggested that CAR might reflect increased inflammatory status in such neurological conditions. ${ }^{17-19}$ In a recent study, the association between depression severity and CAR has been examined in patients diagnosed lung cancer. ${ }^{20}$ However, to date, CAR has not been studied in schizophrenia or other psychotic disorders.

Peripheral biomarkers of inflammation derived from complete blood counts (CBC) have been investigated in numerous studies in recent years and suggested to reflect the pathogenesis of schizophrenia. The neutrophil/lymphocyte ratio (NLR), platelet/lymphocyte ratio (PLR), monocyte/lymphocyte ratio (MLR), red-cell distribution width (RDW), and mean platelet volume (MPV) are inexpensive and reproducible biomarkers of inflammation and easily measured in a CBC. Several lines of evidence showed elevated levels of these markers in both mood disorders ${ }^{21,22}$ and schizophrenia. ${ }^{23}$ However, studies are fraught with conflicting results, which are unable to unravel the complexity of the immunologic architecture of the clinical stages of schizophrenia, and raised questions as to whether acute relapse is associated with higher inflammatory tone and if such biomarkers are trait- or state-related. ${ }^{24}$

In this study, alongside CAR and CBC-derived biomarkers of inflammation, we also focused on the neutrophil/albumin ratio (NAR), which has recently only been studied in patients with cancer, because higher NAR indicates an enhanced inflammatory status that worsens cancer prognosis and treatment response. ${ }^{25}$ Likewise CAR, NAR has not been examined in psychiatric disorders to date. In this study, our aims were as follows: 1) to examine CAR and NAR as novel peripheral inflammatory markers in patients with both acutely exacerbated and remitted schizophrenia, and to compare them between patient and control groups; 2) to reveal whether peripheral inflammatory markers discriminated patients and controls and whether these markers varied between patients with acute exacerbation and remission; and 3) to determine optimum cutoff levels of CAR and NAR for the diagnosis of schizophrenia. We hypothesized that 1) regardless of being in relapse or remission, patients with schizophrenia have higher levels of
CAR and NAR compared with healthy subjects, 2) CAR, NAR, and other inflammatory markers do not differ between relapsed and remitted schizophrenia groups, suggesting that inflammation is rather a trait marker for schizophrenia; and 3) there are positive correlations between CAR, NAR, and CBCderived inflammatory markers. Thus, the results of this study could provide us to measure the inflammatory status between exacerbated and remitted patients in schizophrenia and specify a cut-off levels of CAR and NAR for the diagnosis of schizophrenia, which may be beneficial in clinical practice.

\section{METHODS}

\section{Data source and selection criteria}

This cross-sectional retrospective study consecutively included data of patients who were admitted to the outpatient unit or the inpatient clinic in the Bakirkoy Prof Mazhar Osman Training and Research Hospital for Psychiatry, Neurology, and Neurosurgery within a six-month period between September 15th, 2018, and March 15th, 2019, and who were diagnosed as having schizophrenia based on the International Classification of Diseases-10 (ICD-10). Patient data, which were retrieved anonymously by the researchers upon the permission of the institute board, was extracted from the electronic medical records of the hospital without any accessible personal identifying information of the patients except their hospital registration number. Inclusion criteria for patients were as follows: 1) age $18-65$ years; 2) being admitted or hospitalized with a primary diagnosis of schizophrenia (ICD-10 codes between F20.0-F20.9). Exclusion criteria for schizophrenia patients were as follows: presence of a comorbid psychiatric disorder, presence of a systemic disease that may influence inflammatory status such as diabetes mellitus, hepatic or renal failure, hypertension, acute infection, acute or chronic immuno-inflammatory disease or pregnancy, obesity or being underweight (body-mass index $>29.9 \mathrm{~kg} / \mathrm{m}^{2}$ or $<18.5 \mathrm{~kg} / \mathrm{m}^{2}$, respectively), heavy smoking (20>cigarettes per day), being under an anti-inflammatory or immunosuppressive medication or psychotropic medication other than antipsychotics, documented laboratory findings of liver or renal pathology, nutritional deficiency of vitamin B12 or folate and iron-deficiency anemia, and not having a laboratory screening at the admission. A comparison group of healthy controls consisted of 445 individuals who visited our outpatient unit for purposes of pre-employment health check-ups or employee medical examinations, coded with ICD-10 Z00.00 (encounter for general adult medical examination without abnormal findings), aged 18-65 years, and without any previous psychiatric or medical diagnosis coded and any current medication in the hospital electronic database. 


\section{Variables and measurements}

After the eligibility evaluation of the data for inclusion, the patients' electronic and hard-copy files, which were provided with patients' hospital registration number, were reviewed. According to the hospital protocol, blood sampling is performed in the first $24 \mathrm{~h}$ of hospitalization or on admission of the outpatient unit using a standard venipuncture technique from antecubital veins. Preliminarily, data of 1,498 patients who attended the outpatient unit or were admitted to the inpatient clinic within the designated time frame were screened. Two hundred sixty-seven patients were excluded due to the absence of blood screening, 254 patients were excluded due to presence of psychiatric comorbidity including alcohol and substance use disorders, and 157 patients were excluded due to a documented systemic illness, major pathology in laboratory results, or being under an anti-inflammatory medication. One hundred and two patients were excluded due to usage of other psychotropic medications including antidepressants and benzodiazepines which is confirmed through the electronic prescription database. Six hundred eighteen patients were included in the study and their files were reviewed. The patients were assigned to two groups as schizophrenia-acute exacerbation (AE) and schizophrenia-remission (R). Schizophrenia-AE $(n=439)$ defined patients with a psychiatric emergency admission with an acute relapse or following the first $24 \mathrm{~h}$ of acute psychiatric hospitalization, and schizophrenia- $\mathrm{R}(\mathrm{n}=179)$ represented patients who were admitted to the outpatient setting for a routine follow-up or who gave pre-discharge blood sampling. Remission was determined by clinical examination by a senior psychiatrist during outpatient follow-up or at predischarge evaluation, and systematic operational remission criteria based on the specific items of Positive and Negative Syndrome Scale (PANSS) which is conceptualized by Andreasen et al., ${ }^{26}$ were used to confirm a clinical remission. Age, sex, and laboratory results of all individuals and clinical variables including illness duration, number of hospital stays, and total duration of hospital stays of patients were recorded anonymously. Because the data of the individuals were retrieved anonymously and file review was made retrospectively by the researchers, patient informed consent is not applicable. All procedures performed in this retrospective file review study involving human participants were in accordance with the 1964 Helsinki declaration and its later amendments or comparable ethical standards. The study was approved by the Local Ethics Committee (IRB: 03.05.2016-2016/523).

\section{Statistical analysis}

All statistical analyses were performed using the IBM Statistical Package for the Social Sciences (SPSS) for Mac OS, Version 23.0 software (IBM Corp., Armonk, NY, USA). A chi-square test and an independent sample t-test were used for comparisons of categorical and parametric variables between the patient and the control groups. For the comparison of inflammatory markers amongst the schizophrenia$\mathrm{AE}$, schizophrenia-R, and control group, one-way analysis of variance (ANOVA) was used, and Tukey's honestly significant difference (HSD) test was performed for post-hoc analysis of pairwise comparisons. Pearson's correlation test was performed to analyze associations between peripheral inflammatory markers with each other and with clinical characteristics. Receiver operating characteristic (ROC) analysis was used to show the use of CAR and NAR in differentiating between patients with schizophrenia and healthy controls. A binomial logistic regression analysis was performed to examine the predictive power of inflammatory markers for the diagnosis of schizophrenia. A p-value of less than 0.05 was considered statistically significant.

\section{RESULTS}

\section{Descriptive variables}

Descriptive characteristics of the study population are presented in Table 1 . The study population consisted of 533 males (50.14\%) and 530 females (49.86\%). Males/Females (M/F) ratio for study groups was 310/308 for patients and 223/222 for controls. There were no differences between the patient and control groups with regard to sex $\left(\mathrm{M} / \mathrm{F}_{\text {patient }}=1.006 ; \mathrm{M} / \mathrm{F}_{\text {control }}=1.004\right.$, $\chi^{2}=0.00, \mathrm{p}=0.98$ ); however, age was higher in the patient group compared with the healthy controls $\left(\mu_{\text {patient }} \pm \mathrm{SD}_{\text {patient }}=39.68 \pm\right.$ $\left.10.56 ; \mu_{\text {control }} \pm \mathrm{SD}_{\text {control }}=31.22+9.69, \mathrm{t}=13.515, \mathrm{p}<0.001\right)$. Within the schizophrenia group, 439 had acutely exacerbations and 179 were in remission. The duration of illness $(t=1.928, p=0.54)$ and number of hospital stays $(\mathrm{t}=-0.601, \mathrm{p}=0.54)$ did not differ between the patient groups, whereas the total duration of hospital stays of patients in remission was longer than was for patients with acute exacerbations $(\mathrm{t}=2.179, \mathrm{p}=0.03)$.

\section{Comparison of inflammatory markers between the patient groups and control subjects}

An independent sample t-test showed that the inflammatory markers; CAR $(\mathrm{t}=16.316, \mathrm{p}<0.001), \mathrm{NAR}(\mathrm{t}=13.487$, $\mathrm{p}<0.001)$, NLR $(\mathrm{t}=9.3, \mathrm{p}<0.001), \operatorname{PLR}(\mathrm{t}=5.256, \mathrm{p}<0.001)$, MLR ( $\mathrm{t}=3.21, \mathrm{p}=0.001)$, RDW $(\mathrm{t}=4.064, \mathrm{p}<0.001)$, and MPV $(\mathrm{t}=3.568, \mathrm{p}<0.001)$ were significantly higher in patients with schizophrenia than in control subjects. The results of oneway ANOVA revealed that there were statistically significant differences between the schizophrenia-AE, schizophrenia- $R$, and control groups in terms of CAR $(\mathrm{F}=97.895, \mathrm{p}<0.001)$, NAR $(\mathrm{F}=88.940, \mathrm{p}<0.001), \mathrm{NLR}(\mathrm{F}=39.471, \mathrm{p}<0.001)$, PLR $(\mathrm{F}=11.969, \mathrm{p}<0.001), \operatorname{MLR}(\mathrm{F}=6.411, \mathrm{p}=0.002)$, RDW 
Table 1. Descriptive variables of the study groups

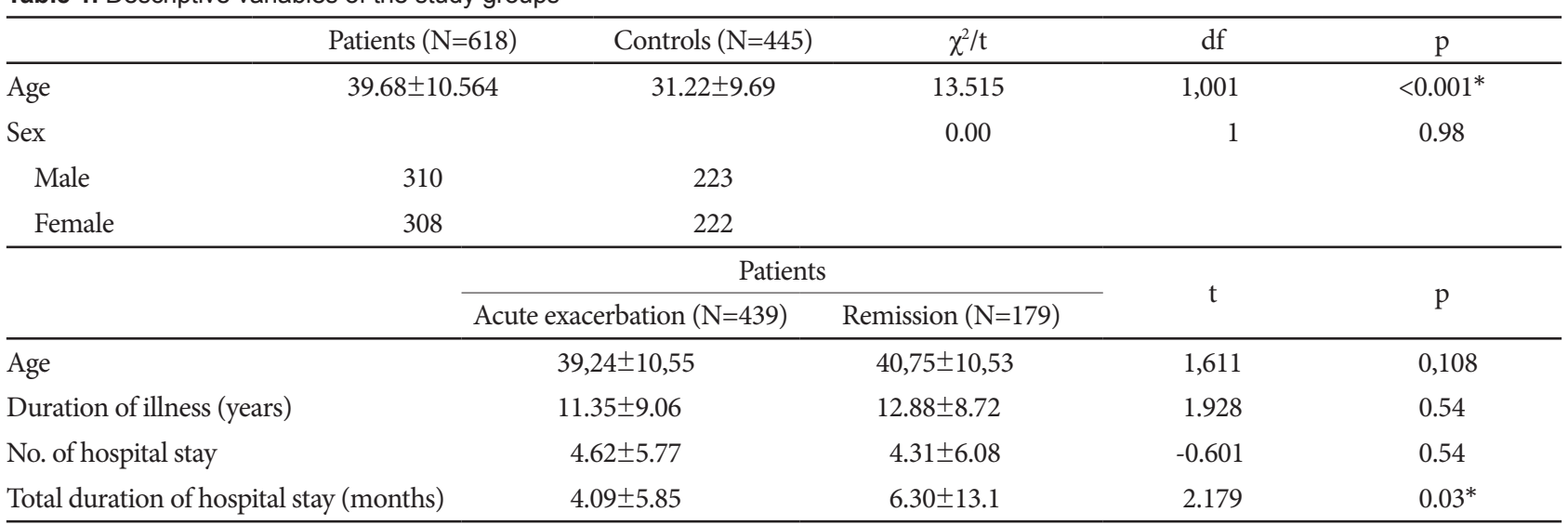

${ }^{*} \mathrm{p}<0.05$ statistically significant. $\chi^{2}$ : chi-square, t: Student's t-test

Table 2. Comparison of peripheral inflammatory markers between the groups

\begin{tabular}{|c|c|c|c|c|c|c|c|c|c|c|}
\hline & \multicolumn{2}{|c|}{ Patients } & \multirow{2}{*}{ Controls } & & \multirow{2}{*}{$\mathrm{p}^{1 *}$} & \multirow{2}{*}{$\mathrm{F}$} & \multirow{2}{*}{$\mathrm{p}^{2 \dagger}$} & \multirow{2}{*}{$\mathrm{p}^{3 \neq}$} & \multirow{2}{*}{$\mathrm{p}^{4 \ddagger}$} & \multirow{2}{*}{$\mathrm{p}^{5 \ddagger}$} \\
\hline & Schizophrenia-AE & Schizophrenia-R & & & & & & & & \\
\hline CAR & $1.54 \pm 2.05$ & $1.34 \pm 1.35$ & $0.23 \pm 0.21$ & 16.316 & $<0.001$ & 97.895 & $<0.001$ & 0.283 & $<0.001$ & $<0.001$ \\
\hline NAR & $1.16 \pm 0.43$ & $1.06 \pm 0.37$ & $0.83 \pm 0.32$ & 13.487 & $<0.001$ & 88.94 & $<0.001$ & 0.007 & $<0.001$ & $<0.001$ \\
\hline NLR & $2.27 \pm 1.41$ & $2.09 \pm 1.17$ & $1.65 \pm 0.6$ & 9.3 & $<0.001$ & 6.411 & $<0.001$ & 0.162 & $<0.001$ & $<0.001$ \\
\hline PLR & $125.03 \pm 58.95$ & $123.27 \pm 62.07$ & $108.93 \pm 36.57$ & 5.256 & 0.001 & 11.969 & $<0.001$ & 0.921 & $<0.001$ & 0.005 \\
\hline MLR & $0.28 \pm 0.13$ & $0.26 \pm 0.10$ & $0.24 \pm 0.20$ & 3.21 & $<0.001$ & 88.94 & 0.002 & 0.253 & 0.001 & 0.496 \\
\hline RDW & $13.93 \pm 2.58$ & $13.95 \pm 2.91$ & $12.92 \pm 5.32$ & 4.064 & $<0.001$ & 8.255 & $<0.001$ & 0.997 & 0.001 & 0.010 \\
\hline MPV & $9.36 \pm 1.94$ & $9.2 \pm 1.72$ & $8.90 \pm 1.86$ & 3.568 & $<0.001$ & 6.804 & 0.001 & 0.615 & 0.001 & 0.158 \\
\hline
\end{tabular}

${ }^{*}$ Student's t-test, ${ }^{\dagger}$ One-way ANOVA, ${ }^{\ddagger}$ Tukey-HSD for ANOVA post-HOC. $\mathrm{p}^{1}$ : patients vs. controls, $\mathrm{p}^{2}$ : schizophrenia-AE vs. schizophrenia-R vs. controls, $\mathrm{p}^{3}$ : schizophrenia-AE vs. schizophrenia-R, $\mathrm{p}^{4}$ : schizophrenia-AE vs. controls, $\mathrm{p}^{5}$ : schizophrenia-R vs. controls, Schizophrenia-AE: acute exacerbation, Schizophrenia-R: remission, CAR: C-reactive protein/ albumin ratio, NAR: neutrophil/albumin ratio, NLR: neutrophil/lymphocyte ratio, PLR: platelet/lymphocyte ratio, MLR: monocyte/lymphocyte ratio, RDW: red-cell distribution width, MPV: mean platelet volume

$(\mathrm{F}=8.255, \mathrm{p}<0.001)$, and MPV $(\mathrm{F}=6.804, \mathrm{p}=0.001)$. Further, post-hoc analysis using Tukey's HSD test was performed and it was found that none of inflammatory markers except NAR $\left(\mu_{\mathrm{AE}} \pm \mathrm{SDAE}=1.16 \pm 0.43 ; \mu_{\mathrm{R}} \pm \mathrm{SD}_{\mathrm{R}}=1.06 \pm 0.37, \mathrm{p}=0.007\right)$ differed between the schizophrenia-AE and schizophrenia- $\mathrm{R}$ groups. All markers were strongly significantly higher in patients with schizophrenia-AE compared with healthy controls $(\mathrm{p}<0.001)$. Additionally, all markers, but MLR $(\mathrm{p}=0.496)$ and MPV ( $p=0.158)$ were significantly higher in patients with schizophrenia- $R$ than in controls $(\mathrm{p}<0.05)$ (Table 2$)$.

\section{Correlation of peripheral inflammatory markers and clinical variables}

The results of Pearson's correlation test, with which we examined bivariate correlations of inflammatory markers in the schizophrenia-AE and schizophrenia-R groups, showed that except CAR-MPV, NAR-MPV, PLR-MPV, and MLRMPV relationships, there were statistically significant and positive correlations between inflammatory markers with each other. CAR, NAR, NLR, PLR, MLR and RDW were not significantly correlated with clinical variables (age, duration of illness, number of hospital stays and total duration of hospital stays). However, MPV was significantly and negatively correlated with the number of hospital stays $(r=-0.099)$ and total duration of hospital stays $(\mathrm{r}=-0.148)$ (Table 3).

\section{Receiver operating characteristics (ROC) for CAR and NAR for the diagnosis of schizophrenia}

The ROC analysis revealed that a CAR value lower than 95\% (95\% CI: 0.863-0.902) and 0.388 as a cut-off, differentiated patients with schizophrenia from healthy controls with a sensitivity of $81 \%$ and a specificity of $81 \%$ (area under the curve (AUC) 0.882, $\mathrm{p}<0.001$ ), and 0.885 as a cut-off value for NAR (95\% CI: 0.712-0.770) differentiated patients with schizophrenia from healthy controls with a sensitivity of $68 \%$ and a specificity of $67.5 \%$ (AUC 0.741, p<0.001) (Figure 1).

\section{The inflammatory marker predictors of the diagnosis of schizophrenia}

The hypothesized relationship between the diagnosis of 
schizophrenia CAR and NAR was further tested in a binomial logistic regression analysis. Gender, CAR, NAR, NLR, PLR, MLR, RDW, and MPV were entered in the first step of the

Table 3. Pearson correlation test coefficients between inflammatory markers and clinical variables

\begin{tabular}{lcccc}
\hline & Age & $\begin{array}{c}\text { Duration of } \\
\text { illness (yrs.) }\end{array}$ & $\begin{array}{c}\text { No. of } \\
\text { hospital stay }\end{array}$ & $\begin{array}{c}\text { Total duration } \\
\text { of hospital } \\
\text { stay (mos.) }\end{array}$ \\
\hline CAR $^{\text {a }}$ & $0.084^{*}$ & 0.071 & 0.021 & -0.001 \\
NAR $^{\mathrm{a}}$ & 0.052 & 0.043 & 0.042 & -0.002 \\
$\mathrm{NLR}^{\mathrm{b}}$ & 0.038 & 0.032 & -0.017 & -0.026 \\
$\mathrm{PLR}^{\mathrm{a}}$ & $0.127^{\dagger}$ & 0.043 & -0.018 & -0.032 \\
MLR $^{\mathrm{c}}$ & 0.034 & 0.013 & 0.009 & -0.017 \\
RDW $^{\mathrm{c}}$ & 0.024 & 0.007 & -0.011 & -0.067 \\
MPV $^{\mathrm{a}}$ & -0.015 & -0.056 & $-0.099^{*}$ & $-0.148^{\dagger}$ \\
\hline
\end{tabular}

${ }^{a}$ Statistical significance was not observed for CAR-MPV, NARMPV, and PLR-MPV correlations ( $p>0.05$ ), while observed for other bivariate correlations; ${ }^{b}$ significantly correlated with all other inflammatory markers $(\mathrm{p}<0.05)$; ${ }^{\mathrm{C}} \mathrm{RDW}-\mathrm{MLR}$ is not significantly correlated; significant correlation existed with other markers $(\mathrm{p}<0.05)$, among all schizophrenia patients. $\mathrm{r}$ : Pearson correlation test coefficient. *correlation is significant at the 0.05 level (twotailed), ${ }^{\dagger}$ correlation is significant at the 0.01 level (two-tailed). CAR: C-reactive protein/albumin ratio, NAR: neutrophil/albumin ratio, NLR: neutrophil/lymphocyte ratio, PLR: platelet/lymphocyte ratio, MLR: monocyte/lymphocyte ratio, RDW: red-cell distribution width, MPV: mean platelet volume

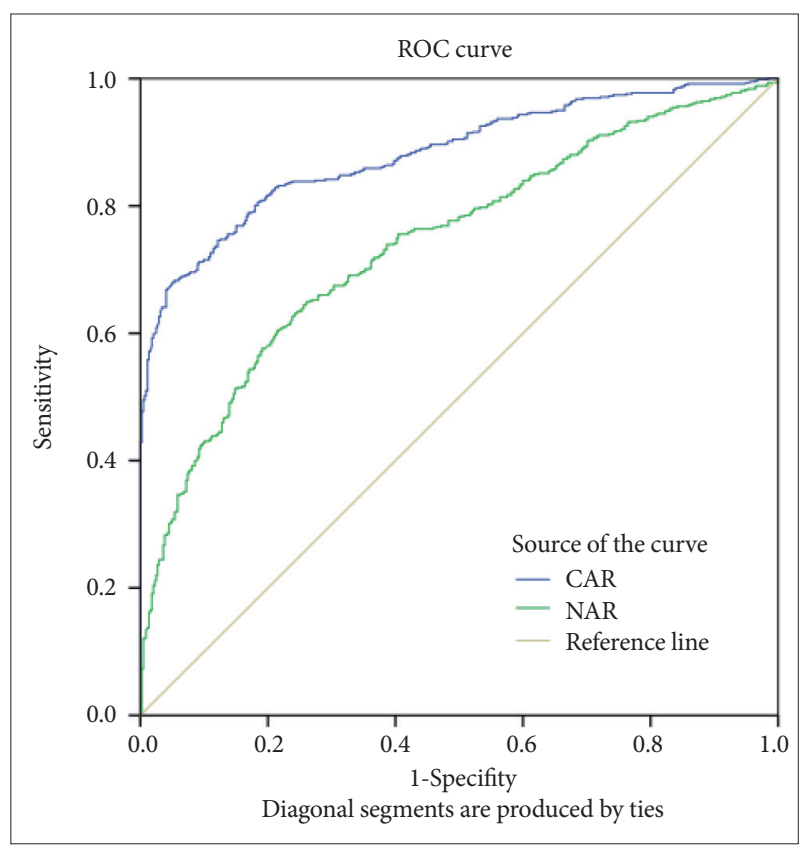

Figure 1. Receiver operating characteristic (ROC) curves for the diagnostic ability of CAR and NAR (patient vs. control). ROC curves for CAR and NAR values for the diagnosis of schizophrenia. CAR: AUC 0.882 (95\% $\mathrm{Cl}=0.863$ to 0.902$), p<0.001$; NAR: AUC 0.741 ( $95 \% \mathrm{Cl}=0.712$ to 0.770$), \mathrm{p}<0.001$. AUC: area under the ROC curve, CAR: C-reactive protein/albumin ratio, NAR: neutrophil/albumin ratio. analysis. We decided not to test the predictive value of age because the mean age of patients was significantly higher than that of controls, which implied that age would have a substantial effect on the diagnosis of schizophrenia. The results of the regression analysis are displayed in Table 4 . The logistic regression analysis indicated that CAR $(\mathrm{p}<0.001)$, NAR $(\mathrm{p}=0.001)$, and MPV ( $\mathrm{p}=0.001$ ) were significant predictors of diagnosis.

\section{DISCUSSION}

The principal findings of the present study were that CAR, NAR, NLR, PLR, MLR, RDW, and MPV were peripheral inflammatory markers that were increased in a relatively large sample of patients with schizophrenia compared with healthy controls. Despite CAR values having been recently reported in patients with Parkinson disease, ${ }^{19}$ restless leg syndrome, ${ }^{18}$ and migraine,${ }^{17}$ this is the first study to examine CAR and NAR in patients with with schizophrenia.

In terms of the other inflammatory markers we studied, our findings were consistent with previous research. Two recent meta-analyses demonstrated that CBC-derived biomarkers of inflammation including NLR, PLR, and MLR were higher in patients with schizophrenia than in healthy subjects; ${ }^{23,27}$ however, they reported no significant alterations of these markers between patients with acute and chronic disease.

CRP and albumin screening are readily available, usually within minutes of acute admission, and are often performed automatically as part of an admission profile. Albumin has a myriad of substantial physiologic effects that are essential for normal health. It has been well documented that serum albumin levels are correlated strongly with dietary protein intake; ${ }^{28}$ however, recently, researchers and physicians have appreciated the influence of inflammation on the serum albumin concentration. Given that albumin is a negative acute- phase protein, inflammation leads to a decrease in serum albumin levels because of several factors, including reduced synthesis, increased catabolism, and translocation of albumin to extravascular pools from vessels. ${ }^{29,30}$ Proinflammatory cytokines including tumor necrosis factor (TNF)-alpha and interleukin-6 (IL-6) are thought to induce albumin catabolism, ${ }^{31}$ and TNF-alpha has been shown to inhibit albumin gene expression in a murine model..$^{32}$ The CAR is a novel biochemical marker of systemic inflammatory response and has been associated with poor survival, ${ }^{16,33}$ and treatment response ${ }^{34}$ in various cancer types, inflammatory activity in autoimmune diseases, ${ }^{35,36}$ and sepsis. ${ }^{15}$ CAR has been suggested to be a better inflammatory marker of prognosis than CRP or albumin alone. ${ }^{15}$ In addition, CAR has a weak but significant correlation with NLR; ${ }^{37}$ however, in our study, CAR was strongly correlated with other peripheral inflammatory markers including NLR. 
Table 4. Results of binomial logistic regression for the diagnosis of schizophrenia predictors

\begin{tabular}{|c|c|c|c|c|c|c|c|}
\hline $\begin{array}{l}\text { Variables in } \\
\text { the equation }\end{array}$ & B & S.E. & Wald & df & Sig & $\operatorname{Exp}(B)$ & $\begin{array}{c}\text { 95\% CI for } \\
\operatorname{Exp}(B)\end{array}$ \\
\hline \multicolumn{8}{|l|}{ Step $1^{*}$} \\
\hline Sex (male) & 0.092 & 0.197 & 0.221 & 1 & 0.639 & 1.097 & $0.746-1.613$ \\
\hline CAR & -4.402 & 0.327 & 181.734 & 1 & 0.000 & 0.012 & $0.006-0.023$ \\
\hline NAR & -1.427 & 0.420 & 11.534 & 1 & 0.001 & 0.240 & $0.114^{--} 0.349$ \\
\hline NLR & -0.140 & 0.206 & 0.460 & 1 & 0.497 & 0.870 & $0.581-1.302$ \\
\hline PLR & -0.004 & 0.003 & 1.943 & 1 & 0.163 & 0.996 & $0.990-1.002$ \\
\hline MLR & 0.226 & 0.671 & 0.113 & 1 & 0.737 & 1.253 & $0.337-4.664$ \\
\hline RDW & -0.014 & 0.023 & 0.407 & 1 & 0.523 & 0.986 & $0.943-1.030$ \\
\hline MPV & -0.163 & 0.050 & 10.687 & 1 & 0.001 & 0.850 & $0.771--0.937$ \\
\hline Constant & 5.538 & 0.700 & 62.666 & 1 & 0.000 & 254.223 & \\
\hline
\end{tabular}

*variable (s) entered on Step 1: CAR, NAR, NLR, PLR, MLR, RDW, MPV; dependent variable: diagnosis of schizophrenia. CAR: C-reactive protein/albumin ratio, NAR: neutrophil/albumin ratio, NLR: neutrophil/lymphocyte ratio, PLR: platelet/lymphocyte ratio, MLR: monocyte/ lymphocyte ratio, RDW: red-cell distribution width, MPV: mean platelet volume

We also found that none of the peripheral systemic inflammation markers, except NAR, differed between patients with acute exacerbation and remitted schizophrenia, which may support the notion that inflammation is rather a trait-related phenomenon in schizophrenia. Chronic inflammation usually includes a lower grade inflammatory response and it plays a role in the pathophysiology of many neuropsychiatric diseases. ${ }^{38}$ Despite there being a long-standing debate as to the extent to which systemic inflammation resembles neuroinflammation, it is now recognized that the brain is not entirely isolated from the peripheral inflammatory response; cytokines and other circulating inflammatory mediators can reach and influence the brain through several complex mechanisms that interact with the blood-brain barrier. ${ }^{39}$ The blood concentrations of some inflammatory molecules appear to vary with the clinical status of schizophrenia; IL-1-beta, IL-6, and transforming growth factor (TGF)-beta were found to be state-related, whereas IL-12, interferon (IFN)-gamma, and TNF-alpha were seen to be trait markers. ${ }^{40}$ However, the term 'state' is not clear enough to define whether it refers to relapse or clinical severity. For instance, an increase in IL-6, a so-called state marker, is positively associated with both positive and negative symptoms as assessed using the PANSS; however, such a clinical condition is not necessarily required to meet a clinical relapse threshold. ${ }^{41}$ It is suggested that greater systemic inflammation generates a tendency towards a full-blown psychosis among individuals at clinical high-risk for psychosis. ${ }^{42}$ First-episode psychosis is closely associated with increased inflammatory markers, despite the fact that such an association has not been explicitly observed in patients with schizophrenia with a clinical relapse. ${ }^{43,44}$ Disturbances in blood cell counts, increased CBC-derived inflammatory parameters and elevated levels of circulating inflammatory mediators including CRP, were reported in schizophrenia independent of clinical course. ${ }^{45,46}$ On the other hand, several studies showed that more severe psychopathology and clinical symptoms in schizophrenia could be related with increased peripheral inflammatory markers. ${ }^{4-49}$ In light of previous findings, one can postulate that it remains inconclusive as to whether an acute relapse is directly associated with increased inflammatory signaling. In fact, increased inflammatory tonus, whether state- or trait-related, has been suggested to be involved in the pathogenesis of schizophrenia through dopaminergic and glutamatergic transmission alterations. ${ }^{2}$ Our findings are consistent with the insight that inflammation is not directly associated with clinical relapse; it rather reflects a pathophysiologic process that may be related to the occurrence of schizophrenia. In our opinion, this may be because of that as the duration of the disease gets longer, the inflammatory status tends to remain stable despite exacerbations. However, we should admit that, the difference in NAR values between the acute exacerbation and remission groups is worthy of further research because a rational explanation is needed.

We found no association between clinical characteristics (illness duration, number of hospitalizations, and total duration of hospital stays) and inflammatory markers, except for a negative correlation between MPV and number of hospital stays and total duration of hospital stays. Miller and Goldsmith $^{42}$ stated that the chronicity of schizophrenia might be associated with increased inflammatory markers. However, they also reported that newly-diagnosed first-episode psychosis was also linked with heightened inflammatory markers. Anti-inflammatory agents are more efficacious in first-episode psychosis, but not in the latter episodes, ${ }^{50}$ which supports the idea that anti-inflammatory therapy would decelerate the clinical progression of the disorder at the early stage, but may not succeed in treating acute exacerbations of schizo- 
phrenia. There is also wide recognition that longer duration of disease is associated with metabolic disturbances, poor prognosis, and worse treatment response; ${ }^{51}$ however, in our opinion, extended illness duration may be related with these features through different mechanisms other than increased inflammation in schizophrenia. Furthermore, we only found two of seven markers correlated with age, which has already been found positively correlated with inflammation in the general population. ${ }^{52}$ Our results confirm prior findings that illness duration, ${ }^{47,53}$ and the number of hospitalizations ${ }^{24,54}$ have not been clearly associated with peripheral inflammatory markers. As inpatient admission is mainly required for an acute exacerbation of patients in our hospital, the data of the number of hospital stays may reflect the number of acute relapses of the patients. These findings may indicate that increased inflammatory markers should not be solely attributed to age, illness duration or more relapses in schizophrenia.

We constructed ROC curves to determine optimum cutoff levels of CAR and NAR for the diagnosis schizophrenia. The cut-off value of CAR found in our study was 0.388 with a sensitivity of $81 \%$ and a specificity of $81 \%$, and 0.885 as a cut-off value for NAR had lower sensitivity and specificity. To date, few studies have reported cut-off levels of peripheral inflammatory biomarkers such as NLR, ${ }^{55} \mathrm{IL}-6,{ }^{56}$ and the high sensitivity-CRP/IL-10 ratio; ${ }^{57}$ however; insufficient power of sensitivity and/or specificity for these markers were denoted. Despite there still existing copious room for research to appreciate its diagnostic use, our finding suggests that CAR, as an inflammatory biomarker, may be used to support the diagnosis of schizophrenia.

According to the logistic regression analyses, CAR, NAR, and MPV were significantly predictive of a diagnosis of schizophrenia which are novel findings. In the study of Wysokiński and Szczepocka, ${ }^{58}$ logistic regression analysis failed to demonstrate a significant predictive value of MPV for the diagnosis of schizophrenia, and the predictive values of CAR and NAR for schizophrenia have not yet been studied. Our findings suggest that a combination of inflammatory markers including CAR and NAR could be used to reflect the increased inflammatory status in schizophrenia.

We have to mention several limitations about the study. First, despite there being a line of evidence that demonstrates abnormal inflammatory parameters in drug-naive patients with firstepisode psychosis, ${ }^{59}$ antipsychotics increase the risk of metabolic conditions, which are associated with inflammation, as such we cannot rule out antipsychotic medication's influence on inflammatory markers. Second, age, a possible confounding factor, significantly differed between the groups which may be a result of the consecutive nature of the data acquisition in the study. It is has been suggested that advanced age is associated with chronic low grade inflammation and the number of people affected by age-related diseases including cardiovascular diseases, diabetes mellitus, cancer and dementia which are closely associated with inflammation, increases with age in both men and women. ${ }^{60}$ With this regard, not matching group ages may not be considered a major limitation in terms of ageinflammation interplay because we have already excluded the patients with age-related diseases. Besides, regression analyses in recent studies revealed significant predictive values of inflammatory biomarkers for psychotic disorders, although the mean ages of the patient and control groups were significantly different. ${ }^{45}$ However, we should disclose that mean age differences between our study groups might have an impact on serum levels of inflammatory markers. Third, despite we excluded obesity or being underweight and heavy smoking, BMI and smoking status of the patients might have affected our results. Finally, we did not examine relationship between psychopathological features and inflammatory markers.

In conclusion, this cross-sectional retrospective study showed that, except NAR, peripheral inflammatory markers did not significantly vary between patients with relapse and remission, suggesting that inflammation is rather a trait marker for schizophrenia. Additionally, we brought CAR and NAR as peripheral trait biomarkers, which may reflect enhanced inflammatory signalling in schizophrenia, to the relevant literature. On the other hand, both biomarkers should be further examined in larger patient groups, including their relationships with clinical symptoms and psychopathology. Yet, it is largely unknown as to whether peripheral inflammatory markers reflect or potentially have an impact on the prognosis and course of schizophrenia. It seems key to differentiate inflammation, either as a trait or state feature for schizophrenia, which is increasingly recognised as a pleiotropic disorder. Indeed, much more comprehensive and well-designed biomarker studies are warranted to yield an invaluable insight for characterizing inflammatory markers, whether they reflect the pathogenesis, or the clinical status of schizophrenia, or both, and for developing new potential diagnostic and advanced intervention strategies to reduce risk, treat, and improve quality of life in schizophrenia.

\section{Acknowledgments}

The authors received no specific grant from any funding agency, commercial or not-for-profit sectors for the research, authorship, and/or publication of this article.

\section{Conflicts of Interest}

The authors have no potential conflicts of interest to disclose.

\section{Author Contributions}

Conceptualization and study design: Yasin Hasan Balcioglu, Simge Seren Kirlioglu. Data curation: Simge Seren Kirlioglu, Yasin Hasan Balcioglu. For- 
mal analysis: Yasin Hasan Balcioglu. Investigation: Simge Seren Kirlioglu. Methodology: Yasin Hasan Balcioglu. Project administration: Yasin Hasan Balcioglu, Simge Seren Kirlioglu. Supervision: Yasin Hasan Balcioglu. Validation: Simge Seren Kirlioglu. Visualization: Yasin Hasan Balcioglu. Writing_original draft: Yasin Hasan Balcioglu. Writing-critical review \& editing: Simge Seren Kirlioglu.

\section{ORCID iDs}

Yasin Hasan Balcioglu

https://orcid.org/0000-0002-1336-1724

Simge Seren Kirlioglu

https://orcid.org/0000-0001-9778-6617

\section{REFERENCES}

1. Strous RD, Shoenfeld Y. Schizophrenia, autoimmunity and immune system dysregulation: A comprehensive model updated and revisited. J Autoimmun 2006;27:71-80.

2. Müller N, Weidinger E, Leitner B, Schwarz MJ. The role of inflammation in schizophrenia. Front Neurosci 2015;9:372.

3. Potvin S, Stip E, Sepehry AA, Gendron A, Bah R, Kouassi E. Inflammatory cytokine alterations in schizophrenia: a systematic quantitative review. Biol Psychiatry 2008;63:801-808.

4. Saetre P, Emilsson L, Axelsson E, Kreuger J, Lindholm E, Jazin E. Inflammation-related genes up-regulated in schizophrenia brains. BMC Psychiatry 2007;7:46.

5. Trépanier MO, Hopperton KE, Mizrahi R, Mechawar N, Bazinet RP. Postmortem evidence of cerebral inflammation in schizophrenia: a systematic review. Mol Psychiatry 2016;21:1009-1026.

6. Eaton WW, Byrne M, Ewald H, Mors O, Chen CY, Agerbo E, et al. Association of schizophrenia and autoimmune diseases: linkage of Danish national registers. Am J Psychiatry 2006;163:521-528.

7. Khandaker GM, Cousins L, Deakin J, Lennox BR, Yolken R, Jones PB. Inflammation and immunity in schizophrenia: implications for pathophysiology and treatment. Lancet Psychiatry 2015;2:258-270.

8. Leza JC, Bueno B, Bioque M, Arango C, Parellada M, Do K, et al. Inflammation in schizophrenia: a question of balance. Neurosci Biobehav Rev 2015;55:612-626.

9. Kushner I. The phenomenon of the acute phase response. Ann N Y Acad Sci 1982;389:39-48.

10. Ridker PM, Hennekens CH, Buring JE, Rifai N. C-reactive protein and other markers of inflammation in the prediction of cardiovascular disease in women. N Engl J Med 2002;342:836-843.

11. Ritchie RF, Palomaki GE, Neveux LM, Navolotskaia O, Ledue TB, Craig WY. Reference distributions for the negative acute-phase serum proteins, albumin, transferrin and transthyretin: a practical, simple and clinically relevant approach in a large cohort. J Clin Lab Anal 1999;13: 273-279.

12. Miller BJ, Culpepper N, Rapaport MH. C-reactive protein levels in schizophrenia: a review and meta-analysis. Clin Schizophr Relat Psychoses 2014;7:223-230.

13. Labad J, Stojanovic-Pérez A, Montalvo I, Solé M, Cabezas Á, Ortega L, et al. Stress biomarkers as predictors of transition to psychosis in atrisk mental states: Roles for cortisol, prolactin and albumin. J Psychiatr Res 2015;60:163-169.

14. Chen S, Xia H Sen, Zhu F, Yin GZ, Qian ZK, Jiang CX, et al. Association between decreased serum albumin levels and depressive symptoms in patients with schizophrenia in a Chinese Han population: A pilot study. Psychiatry Res 2018;270:438-442.

15. Ranzani OT, Zampieri FG, Forte DN, Azevedo LCP, Park M. C-reactive protein/albumin ratio predicts 90 -day mortality of septic patients. PLoS One 2013;8:e59321.

16. Li N, Tian GW, Wang Y, Zhang H, Wang ZH, Li G. Prognostic role of the pretreatment $\mathrm{C}$-reactive protein/albumin ratio in solid cancers: a meta-analysis. Sci Rep 2017;7:41298.

17. Yazar HO, Yazar T, Aygün A, Kaygisiz Ş, Kirbaş D. Evaluation of simple inflammatory blood parameters in patients with migraine. Ir J Med
Sci 2020;189:677-683.

18. Olgun Yazar H, Yazar T, Özdemir S, Kasko Arici Y. Serum C-reactive protein/albumin ratio and restless legs syndrome. Sleep Med 2019;58: 61-65.

19. Yazar T, Yazar HO. Evaluation of C-reactive protein/albumin ratio according to stage in patients with idiopathic parkinson disease. Turk Noroloji Derg 2019;25:123.

20. McFarland DC, Breitbart W, Miller AH, Nelson C. Depression and inflammation in patients with lung cancer: a comparative analysis of acute phase reactant inflammatory markers. Psychosomatics 2020 [Epub ahead of print].

21. Mazza MG, Lucchi S, Tringali AGM, Rossetti A, Botti ER, Clerici M. Neutrophil/lymphocyte ratio and platelet/lymphocyte ratio in mood disorders: a meta-analysis. Prog Neuro-Psychopharmacology Biol Psychiatry 2018;84:229-236.

22. Kirlioglu SS, Balcioglu YH, Kalelioglu T, Erten E, Karamustafalioglu N. Comparison of the complete blood count-derived infl ammatory markers in bipolar patients with manic and mixed episodes. Bratislava Med J 2019;120:195-199.

23. Mazza MG, Lucchi S, Rossetti A, Clerici M. Neutrophil-lymphocyte ratio, monocyte-lymphocyte ratio and platelet-lymphocyte ratio in nonaffective psychosis: a meta-analysis and systematic review. World J Biol Psychiatry 2020;21:326-338.

24. Özdin S, Böke Ö. Neutrophil/lymphocyte, platelet/lymphocyte and monocyte/lymphocyte ratios in different stages of schizophrenia. Psychiatry Res 2019;271:131-135.

25. Tingle SJ, Severs GR, Goodfellow M, Moir JA, White SA. NARCA: a novel prognostic scoring system using neutrophil-albumin ratio and Ca19-9 to predict overall survival in palliative pancreatic cancer. J Surg Oncol 2018;118:680-686.

26. Andreasen NC, Carpenter WT, Kane JM, Lasser RA, Marder SR, Weinberger DR. Remission in schizophrenia: proposed criteria and rationale for consensus. Am J Psychiatry 2005;162:441-449.

27. Karageorgiou V, Milas GP, Michopoulos I. Neutrophil-to-lymphocyte ratio in schizophrenia: A systematic review and meta-analysis. Schizophr Res 2019;206:4-12.

28. Kirsch R, Frith L, Black E, Hoffenberg R. Regulation of albumin synthesis and catabolism by alteration of dietary protein. Nature 1968;217:578-579.

29. Don BR, Kaysen G. Serum albumin: relationship to inflammation and nutrition. Semin Dial 2004;17:432-437.

30. Artigas A, Wernerman J, Arroyo V, Vincent JL, Levy M. Role of albumin in diseases associated with severe systemic inflammation: Pathophysiologic and clinical evidence in sepsis and in decompensated cirrhosis. J Crit Care 2016;33:62-70.

31. Kaysen GA, Chertow GM, Adhikarla R, Young B, Ronco C, Levin NW. Inflammation and dietary protein intake exert competing effects on serum albumin and creatinine in hemodialysis patients. Kidney Int 2001;60:333-340.

32. Brenner DA, Buck M, Feitelberg SP, Chojkier M. Tumor necrosis factor- $\alpha$ inhibits albumin gene expression in a murine model of cachexia. J Clin Invest 1990;85:248-255.

33. Xu HJ, Ma Y, Deng F, Ju WB, Sun XY, Wang H. The prognostic value of C-reactive protein/albumin ratio in human malignancies: an updated meta-analysis. Onco Targets Ther 2017;10:3059-3070.

34. Kinoshita A, Onoda H, Imai N, Iwaku A, Oishi M, Tanaka K, et al. The C-reactive protein/albumin ratio, a novel inflammation-based prognostic score, predicts outcomes in patients with hepatocellular carcinoma. Ann Surg Oncol 2015;22:803-810.

35. Qin G, Tu J, Liu L, Luo L, Wu J, Tao L, et al. Serum albumin and C-reactive protein/albumin ratio are useful biomarkers of Crohn's disease activity. Med Sci Monit 2016;22:4393-4400.

36. Seringec Akkececi N, Yildirim Cetin G, Gogebakan H, Acipayam C. The C-reactive protein/albumin ratio and complete blood count parameters as indicators of disease activity in patients with Takayasu arteritis. Med Sci Monit 2019;25:1401-1409. 
37. Saito H, Kono Y, Murakami Y, Shishido Y, Kuroda H, Matsunaga T, et al. Prognostic significance of the preoperative ratio of C-reactive protein to albumin and neutrophil-lymphocyte ratio in gastric cancer patients. World J Surg 2018;42:1819-1825.

38. Perry VH. Contribution of systemic inflammation to chronic neurodegeneration. Acta Neuropathol 2010;120:277-286.

39. Becher B, Spath S, Goverman J. Cytokine networks in neuroinflammation. Nat Rev Immunol 2017;17:49-59.

40. Kirkpatrick B, Miller BJ. Inflammation and schizophrenia. Schizophr Bull 2013;39:1174-1179.

41. Stojanovic A, Martorell L, Montalvo I, Ortega L, Monseny R, Vilella E, et al. Increased serum interleukin-6 levels in early stages of psychosis: associations with at-risk mental states and the severity of psychotic symptoms. Psychoneuroendocrinology 2014;41:23-32.

42. Miller BJ, Goldsmith DR. Inflammatory biomarkers in schizophrenia: implications for heterogeneity and neurobiology. Biomarkers Neuropsychiatry 2019;1:1-29.

43. Borovcanin M, Jovanovic I, Radosavljevic G, Djukic Dejanovic S, Bankovic D, Arsenijevic N, et al. Elevated serum level of type- 2 cytokine and low IL-17 in first episode psychosis and schizophrenia in relapse. J Psychiatr Res 2012;46:1421-1426.

44. Borovcanin M, Jovanovic I, Radosavljevic G, Djukic Dejanovic S, Stefanovic V, Arsenijevic N, et al. Antipsychotics can modulate the cytokine profile in schizophrenia: Attenuation of the type-2 inflammatory response. Schizophr Res 2013;147:103-109.

45. Miller BJ, Mellor A, Buckley P. Total and differential white blood cell counts, high-sensitivity C-reactive protein, and the metabolic syndrome in non-affective psychoses. Brain Behav Immun 2013;31:82-89.

46. Garcia-Rizo C, Casanovas M, Fernandez-Egea E, Oliveira C, Meseguer A, Cabrera B, et al. Blood cell count in antipsychotic-naive patients with non-affective psychosis. Early Interv Psychiatry 2019;13:95-100.

47. Fan X, Pristach C, Liu EY, Freudenreich O, Henderson DC, Goff DC. Elevated serum levels of C-reactive protein are associated with more severe psychopathology in a subgroup of patients with schizophrenia. Psychiatry Res 2007;149:267-271.

48. Fan X, Liu EY, Freudenreich O, Park JH, Liu D, Wang J, et al. Higher white blood cell counts are associated with an increased risk for metabolic syndrome and more severe psychopathology in non-diabetic patients with schizophrenia. Schizophr Res 2010;118:211-217.
49. Barzilay R, Lobel T, Krivoy A, Shlosberg D, Weizman A, Katz N. Elevated C-reactive protein levels in schizophrenia inpatients is associated with aggressive behavior. Eur Psychiatry 2016;31:8-12.

50. Müller N, Krause D, Dehning S, Musil R, Schennach-Wolff R, Obermeier $\mathrm{M}$, et al. Celecoxib treatment in an early stage of schizophrenia: Results of a randomized, double-blind, placebo-controlled trial of celecoxib augmentation of amisulpride treatment. Schizophr Res 2010;121:118-124.

51. Heilbronner U, Samara M, Leucht S, Falkai P, Schulze TG. The longitudinal course of schizophrenia across the lifespan: clinical, cognitive, and neurobiological aspects. Harv Rev Psychiatry 2016;24:118-128.

52. Chung HY, Cesari M, Anton S, Marzetti E, Giovannini S, Seo AY, et al. Molecular inflammation: underpinnings of aging and age-related diseases. Ageing Res Rev 2009;8:18-30.

53. Fawzi MH, Fawzi MM, Fawzi MM, Said NS. C-reactive protein serum level in drug-free male Egyptian patients with schizophrenia. Psychiatry Res 2011;190:91-97.

54. Semiz M, Yildirim O, Canan F, Demir S, Hasbek E, Tuman TC, et al. Elevated neutrophil/lymphocyte ratio in patients with schizophrenia. Psychiatr Danub 2014;26:220-225.

55. Yüksel RN, Ertek IE, Dikmen AU, Göka E. High neutrophil-lymphocyte ratio in schizophrenia independent of infectious and metabolic parameters. Nord J Psychiatry 2018;72:336-340.

56. Chase KA, Cone JJ, Rosen C, Sharma RP. The value of interleukin 6 as a peripheral diagnostic marker in schizophrenia. BMC Psychiatry 2016;16:152.

57. Zhang Q, Hong W, Li H, Peng F, Wang F, Li N, et al. Increased ratio of high sensitivity C-reactive protein to interleukin-10 as a potential peripheral biomarker of schizophrenia and aggression. Int J Psychophysiol 2017;114:9-15.

58. Wysokiński A, Szczepocka E. Platelet parameters (PLT, MPV, P-LCR) in patients with schizophrenia, unipolar depression and bipolar disorder. Psychiatry Res 2016;237:238-245.

59. Upthegrove R, Manzanares-Teson N, Barnes NM. Cytokine function in medication-naive first episode psychosis: A systematic review and meta-analysis. Schizophr Res 2014;155:101-108.

60. Rea IM, Gibson DS, McGilligan V, McNerlan SE, Denis Alexander H, Ross OA. Age and age-related diseases: role of inflammation triggers and cytokines. Front Immunol 2018;9:586. 